\title{
Prevalence and Anti-Microbial Susceptibility of Methicillin Resistant Staphylococcus aureus at Moi Teaching and Referral Hospital Eldoret
}

\author{
Christine Akoru1, Robert T. Kuremu², Samson K. Ndege³, A. Obala², James W. Smith4, \\ Marilyn Bartlett ${ }^{4}$ \\ ${ }^{1}$ Moi Teaching and Referral Hospital, Microbiology Department, Eldoret, Kenya \\ ${ }^{2}$ Moi University School of Medicine, Eldoret, Kenya \\ ${ }^{3}$ Moi University School of Public Health, Eldoret, Kenya \\ ${ }^{4}$ Indiana University School of Medicine, Indianapolis, IN, USA \\ Email: cnakoru@gmail.com, robkum2002@gmail.com,ndegekip@yahoo.com, rew.obala@gmail.com, \\ jsmith@slant.com
}

Received 14 November 2015; accepted 15 March 2016; published 18 March 2016

Copyright (C) 2016 by authors and Scientific Research Publishing Inc.

This work is licensed under the Creative Commons Attribution International License (CC BY). http://creativecommons.org/licenses/by/4.0/

(c) (i) 0pen Access

\section{Abstract}

Background: Methicillin-resistant Staphylococcus aureus (MRSA) are pathogens that have major negative impact in all aspects of patient care and are increasingly being recognized as troublesome pathogens in the community. The MRSA are multi-drug resistant bacteria responsible for higher morbidity, mortality and cost of treatment in the affected patients. Research on MRSA antimicrobial susceptibility and resistance in developing countries is limited; hence accurate burden is not certain. Objective: To determine the prevalence and susceptibility pattern of MRSA in $S$. aureus isolates from patients treated in various units at Moi Teaching and Referral Hospital. Study Design: Cross-sectional design was used to conduct the study. Methods and Materials: Staphylococcus aureus stored isolates from patients at various units at Moi Teaching and Referral Hospital (MTRH) were identified by laboratory conventional methods. Methicillin resistant Staphylococcus aureus was determined using cefoxitin and oxacillin, discs and confirmed by Penicillin Binding Protein (PBP2a') latex agglutination test. Susceptibility testing was performed according to the Clinical Laboratory Standards Institute procedures. Control strains used were: ATCC 43300 for Methicillin resistant $S$. aureus (MRSA) strains and ATCC 29213 for methicillin susceptible $S$. aureus (MSSA) strains. Nine anti-microbials (Erythromycin, Gentamycin, Tetracycline, Vancomycin, Rifampin, Linezolid, Ciprofloxacin, Clindamycin and Fucidicacid) were used to test the anti-microbial susceptibility patterns. Control organisms were run along with the isolates for quality Assurance. The data was analyzed using STATA version 11. Categorical variables were summarized as fre- 
quencies and the corresponding percentages. Results were presented using tables and graphs. Results: A total of 107 isolates of $S$. aureus were obtained, of which $39(37 \%)$ were MRSA. Most of the MRSA, $13(33 \%)$ and $7(17 \%)$, were found in pus and tracheal aspirate samples respectively. Majority of the MRSA isolates were from surgical wards and intensive care unit. The MRSA isolates were highly resistant to erythromycin $(92 \% ; 36 / 39)$ and tetracycline $(92 \% 36 / 39)$ and moderately susceptible to linezolid (77\% 30/39), Vancomycin $(75 \% 29 / 39)$ and fucidic acid $(67 \%$ 26/39). A total of $28(74.4 \%)$ MRSA isolates were Clindamycin inducible resistant. Conclusions and Recommendations: This study showed that MRSA is a significant pathogen at MTRH. The MRSA were most detected in pus specimens and least in peritoneal aspirates. Routine Screening of $S$. aureus isolates for MRSA strains at MTRH is therefore imperative. Infection control measures should be put in place to reduce prevalence and prevent the spread of MRSA at MTRH.

\section{Keywords}

\section{MRSA, High Burden Specimens, Wards with High Burden of Isolates and Drug Susceptibility}

\section{Introduction}

Methicillin-resistant Staphylococcus aureus (MRSA) are pathogens that have major negative impact in all aspects of patient care and are increasingly being recognized as troublesome pathogens in the community [1]. The MRSA are multi-drug resistant bacteria responsible for higher morbidity, mortality and cost of treatment in the affected patients [2] [3]. Research on MRSA antimicrobial susceptibility and resistance in developing countries is limited hence accurate burden is not certain [4] [5].

Staphylococcus aureus has the ability to acquire resistance to antimicrobials. Benzyl-Penicillin lost its efficacy to treat Staphylococcus aureus (S. aureus) within 10 years of its introduction because of acquisition of plasmid-encoded $\beta$-lactamse [6]. This was followed by pandemics of S. aureus throughout the late 1950s and early 1960s [7].

Methicillin-resistant S.aureus (MRSA) was first reported in $1961 \mathrm{few}$ years after methicillin was introduced to treat S. aureus infections that were penicillin-resistant [8]. The MRSA harbour additional antimicrobial resistance determinants, which are difficult to treat and are currently the most common cause of nosocomial infections worldwide [9]. The MRSA is considered to have emerged from S. aureus through acquisition of staphylococcal cassette chromosome mec (SCCmec) that carries the mecA gene for methicillin resistance [10]-[12]. Methicillin resistant Staphylococcus aureus is now endemic in most hospitals and healthcare facilities in industrialized countries. Most of MRSA are multi-drug resistant and have been associated with significant mobility as a single infectious agent [13]-[15].

Despite its widespread occurrence worldwide, little information on MRSA prevalence is known in this region. This study hence sought to determine the prevalence at MTRH, a high volume National Referral health facility serving western Kenya, with a population of about 18 million.

\section{Materials and Methods}

Cross-sectional design was used to conduct the study. The study mainly targeted all S. aureus preserved isolates from specimens (Pus, Tracheal Aspirate, urine, Blood, Ascitic Fluid, Cerebral Spinal Fluid, Peritoneal Fluid and Synovial Fluid) of patients attended to in nine (9) different units New born unit (NBU), Burns unit, Surgical wards, Medical wards, pediatric wards, Intensive Care Unit (ICU), Renal unit, Private wards and Out patient (OPD) at Moi Teaching and Referral Hospital from March 2010-December 2011.

The isolates were preserved in cryovials containing two (2) milliliters of Tryptone Soya Broth (Oxoid Ltd, England) with $10 \%$ glycerol and stored at $-20^{\circ} \mathrm{C}$ in storage boxes. Each cryovial in had a unique number for identification and for confidentiality purposes. Each cryovial had a specified location in the storage cryobox and the same was reflected in the manifest forms for easy retrieval during processing. Laboratory numbers were used to identify isolates

The sensitivity culture media (BD Mueller-Hinton-11 Agar) were prepared in-house following manufacturer's 
instructions and were subjected to internal quality control $(Q C)$ using known methicillin-resistant Staphylococcus aureus strains (ATCC 43300) and methicillin susceptible Staphylococcus aureus strains (ATCC 25923). The goal of quality control program was to monitor the precision and accuracy of the procedures and reagents.

The 0.5 McFarland Standards were used as a reference to adjust the turbidity of bacterial suspension in saline so that the number of bacteria was within a given range of approximately $10^{8} \mathrm{CFU} / \mathrm{ml}$ bacterial counts The 0.5 McFarland standards were verified by using a spectrophotometer (Vitex colorimeter, Biomerleux, inc, USA), with a 1-cm light path, and the absorbance at $625 \mathrm{~nm}$.

The 120 preserved isolates were retrieved and thawed. A suspension of the isolates was made and standardized to 0.5 McFarland standard turbidity. Using $1 \mu \mathrm{l}$ loop, a suspension was picked and was inoculated and streaked onto the first $100 \mathrm{~mm}$ Mueller-Hinton agar plate and incubated at $35^{\circ} \mathrm{C}$ for 24 hours. A second suspension of 0.5 McFarland turbidity was made from the 24 hours growth and inoculated directly onto the second Mueller-Hinton $100 \mathrm{~mm}$ plates [16]. The plates were incubated at $35^{\circ} \mathrm{C}$ for 18 - 24 hours. Identification was done according to the laboratory conventional methods.

Methicillin resistance was determined using cefoxitin (30 $\mu \mathrm{g})$ and Oxacillin $(1 \mu \mathrm{g})(\mathrm{BD})$ discs by disc diffusion Method. Zone of inhibition of $\leq 21 \mathrm{~mm}$ was considered resistant and zone of inhibition $\geq 22 \mathrm{~mm}$ was considered susceptible for both oxacillin and cefoxitin [17]. The MRSA screening was confirmed using PBP2a' latex agglutination test (Oxoid) which was used as a gold standard test for MRSA detection. The results were shown by agglutination reaction where presence of agglutination was positive for PBP2a and absence of agglutination was negative. PBP2a positive results were considered MRSA positive and negative results were considered MRSA negative.

Anti-microbial susceptibility testing was performed using Kirby Bauer disc diffusion Method [17]. Anti-microbial discs (BD BBL) used were, tetracycline (30 $\mu \mathrm{g})$, gentamycin $(10 \mu \mathrm{g})$, erythromycin (15 $\mu \mathrm{g})$, rifampin (5 $\mu \mathrm{g})$, ciprofloxacin $(5 \mu \mathrm{g})$, linezolid $(30 \mu \mathrm{g})$, fusidicacid $(10 \mu \mathrm{g})$, clindanycin $(2 \mu \mathrm{g})$, Vancomycin $(30 \mu \mathrm{g})$. The susceptibility discs were spaced $25 \mathrm{~mm}$ away from each other and the plated agar were incubated at $35^{\circ} \mathrm{C}$ for 24 hours. The ATCC control organisms were run along with the tested isolates to confirm the reliability of methicillin-resistance and susceptibility testing. For Methicillin resistant S. aureus (MRSA) strains, ATCC 43300 controls and methicillin susceptible S. aureus (MSSA) ATCC 29213 control strains were used.

The D-test for determining inducible resistance of clindamycin by erythromycin was performed separately from ordinary antimicrobial susceptibility testing, in which erythromycin and clindamycin disks were placed 15 - $26 \mathrm{~mm}$ apart. A truncated or flattening of the zone of inhibition adjacent to the erythromycin disc (referred to as D-zone) indicated inducible clindamycin resistance [17]. A clindamycin zone of inhibition diameter of 14 mm recognized constitutive resistance.

\subsection{Data Analysis}

The data was analyzed using STATA version 11. Categorical variables were summarized as frequencies and the corresponding percentages. Results were presented using tables and graphs.

\subsection{Study Limitations}

Vancomycin susceptibility testing needed to be done using E-test or performing minimum inhibitory testing (mic) which were not available at MTRH. Molecular testing methods (PCR) to confirm mecA genes in MRSA and to determine epidemiologic bacterial strains were unavailable at MTRH however, PBP2a' latex agglutination test was used to determine the presence of mecA gene.

\subsection{Ethical Consideration}

The actual study commenced after approval by Institutional Research and Ethics committee (IREC). Approval Number: 000763.

\section{Results}

Of 120 preserved isolate, only 107 isolates were confirmed as Staphylococcus aureus and all were included in the study. The thirteen (13) isolates of 120, four (4) were coagulase negative and nine (9) did not rejuvenate. 


\subsection{Screening and Confirmatory Tests for MRSA Detection}

The screening tests used were, oxacillin $(1 \mu \mathrm{g})$ and cefoxitin $(30 \mu \mathrm{g})$ discs. PBP2a latex Agglutination test was used as a gold standard and a confirmatory test for MRSA detection. The data obtained in Table 1 below; shows that, a total of 39/107 (36.5\%) of the isolates were found to be MRSA. The oxacillin $(1 \mu \mathrm{g})$ and cefoxitin $(30 \mu \mathrm{g})$ screening tests each had sensitivity of $92.31 \%$, while the specificity of oxacillin $(1 \mu \mathrm{g})$ and cefoxitin $(30 \mu \mathrm{g})$ were $97.06 \%$ and $100 \%$ respectively.

\subsection{Frequency Distribution of Respective Wards Where MRSA Isolates Were Obtained}

Table 2 below shows the respective wards where the specimens were collected. The table shows that, pus samples were collected from all the wards with surgical 19 (35\%) and the burns units 13 (24\%) bearing the highest number of $S$. aureus strains, followed by the medical wards 8 (15\%). The newborn unit (NBU) had $S$. aureus in blood specimens 7 (41\%) and pediatric ward 6 (35\%) respectively. The medical ward had the greatest number of S. aureus in urine specimens 8 (67\%), ICU had the highest number of S. aureus in tracheal aspirates, 7 (88\%) and CSF in medical and NBU each had the least number of $S$. aureus bacteria $3(43 \%)$.

Table 1. Below shows the results of MRSA among 107 rejuvenated stored isolates.

\begin{tabular}{lcccc}
\hline \multirow{2}{*}{ Test } & & \multicolumn{2}{c}{ PBP2a' } & Total \\
\cline { 3 - 4 } & & Positive & Negative & 38 \\
Oesistant & 36 & 2 & 69 \\
Cenacillin $(1 \mu \mathrm{g})$ & Sensitive & 3 & 66 & 107 \\
& Total & 39 & 68 & 36 \\
& Resistant & 36 & 0 & 71 \\
\hline
\end{tabular}

Table 2. Frequency distribution of respective wards where MRSA isolates were obtained.

\begin{tabular}{|c|c|c|c|c|c|c|c|c|c|c|}
\hline \multirow{2}{*}{$\mathrm{n}(\%)$} & \multicolumn{10}{|c|}{ Specimen } \\
\hline & $\mathrm{n}(\%)$ & Ascitic & Blood & ${ }^{*} \mathrm{CSF}$ & Peritoneal & Pus & Tracheal & Synovial & Urine & $\begin{array}{l}\text { Total number } \\
\text { of wards }\end{array}$ \\
\hline \multirow{10}{*}{ Wards } & Burns unit & & & & & $1(7.7)$ & & $1(50)$ & & $2(5.3)$ \\
\hline & ICU & & & & & 2 (15.4) & $6(85.7)$ & & & 8 (20.5) \\
\hline & Medicine & $3(100)$ & $3(75)$ & 1 (33.3) & $1(50)$ & 3 (23.08) & $1(14.3)$ & 0 & $3(60)$ & 15 (38.5) \\
\hline & NBU & & $1(25)$ & $1(33.3)$ & & $1(7.7)$ & & & & $3(7.7)$ \\
\hline & OPD & & & & & & & & & \\
\hline & Peads & & & $1(33.3)$ & & & & & & $1(2.6)$ \\
\hline & Private & & & & & & & $1(50)$ & & $1(2.6)$ \\
\hline & Renal unit & & & & $1(50)$ & & & & $1(20)$ & $2(5.1)$ \\
\hline & Surgical & & & & & $6(46.2)$ & & & $1(20)$ & 7 (17.9) \\
\hline & $\begin{array}{l}\text { Total number } \\
\text { of specimens }\end{array}$ & $3(100)$ & $4(100)$ & $3(100)$ & $2(100)$ & $13(100)$ & $7(100)$ & $2(100)$ & $5(100)$ & 39 (100) \\
\hline
\end{tabular}

"CSF: Cerebral Spinal Fluid, ICU: Intensive Care Unit, NBU: New born Unit, OPD: Out Patient Department, Peads: Pediatrics. 


\subsection{Proportion of MRSA among the 39 Confirmed MRSA Isolates per Specimen Type}

There were a total of 39 (36.5\%) MRSA found in preserved S. aureus isolates whose specimen of origin were shown in Figure 1 below. There was a high burden of MRSA in pus specimen 13/39 (33\%), followed by tracheal aspirates 7/39 (33\%), urine specimen had 5/39 (13\%), synovial fluid and peritoneal had the same proportion 2/39 (5\%) of MRSA respectively.

\subsection{Anti-Microbial Susceptibility}

The 39/107 (35.5\%) of MRSA isolates analyzed were tested against nine (9) commonly used anti-microbial to determine susceptibility or resistance patterns of MRSA. Of the nine anti-microbial, clindamycin was tested separately because of its unique inducible resistance. Figure 2 show high anti-microbial resistance to both erythromycin, tetracycline 36/39 (92\%) followed by rifampin 35/39 (90\%) and gentamycin 30 (77\%). Resistance was also seen in ciprofloxacin 28 (72\%), reduced resistance was seen in Fucidic acid 13 (33\%), Vancomycin 10 (26\%) and linozolid 9 (23\%). This study has shown that linezolid, vancomycin and fusidic acid were the most effective drugs for MRSA treatment, because they had low anti-microbial resistance 9/39 (23\%), 10/39 (25.6\%) and 13/39 (33.3\%), respectively. The results demonstrated moderate anti-microbial susceptibility was demonstrated for ciprofloxacin (8\%) and Gentamycin (23\%).

\subsection{Inducible Clindamycin Resistance}

Inducible Clindamycin resistance was done separately. The study showed that $74 \%$ isolates had clidamycin inducible resistance $13 \%$ of the isolates had constitutive resistance and 13\% were clindamycin susceptible.

\section{Discussions}

The data presented in the study showed that, MRSA is prevalent at Moi Teaching and Referral Hospital. Thirty six percent (36\%) of 107 isolates were MRSA. This figure is higher than those reported in the region for instance; Nairobi (27.7\%) in Kenya and Mulango (31\%) in Uganda [4] [18]. This figure is however way below the Japanese prevalence of $72 \%$ but much higher than the prevalence levels of North Europe where low figures of $1 \%$ have been recorded in Norway, Sweden and Holland [19].

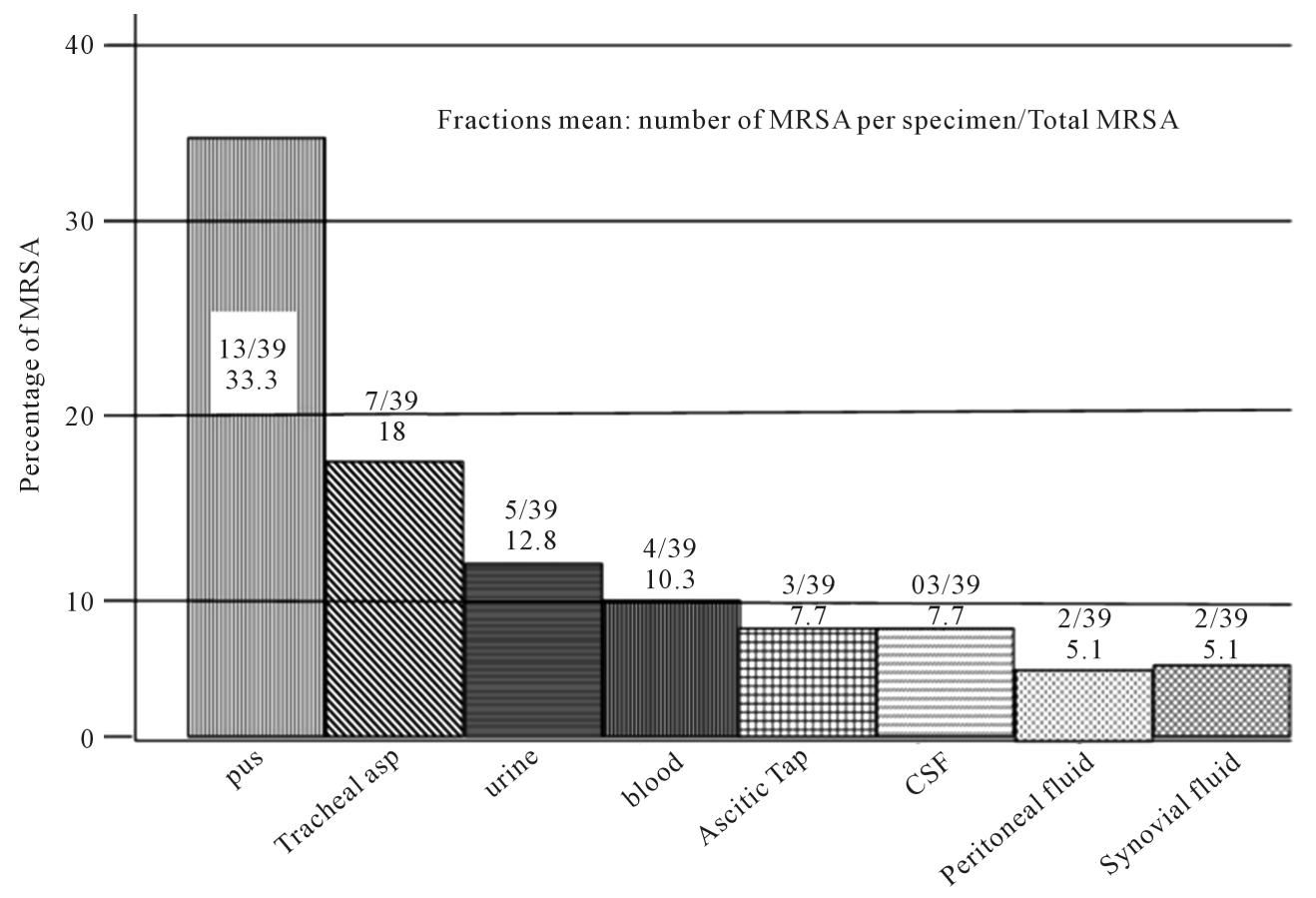

Figure 1. Proportion of MRSA in different specimen type. 
The fractions mean: MRSA resistance to different anti-biogram

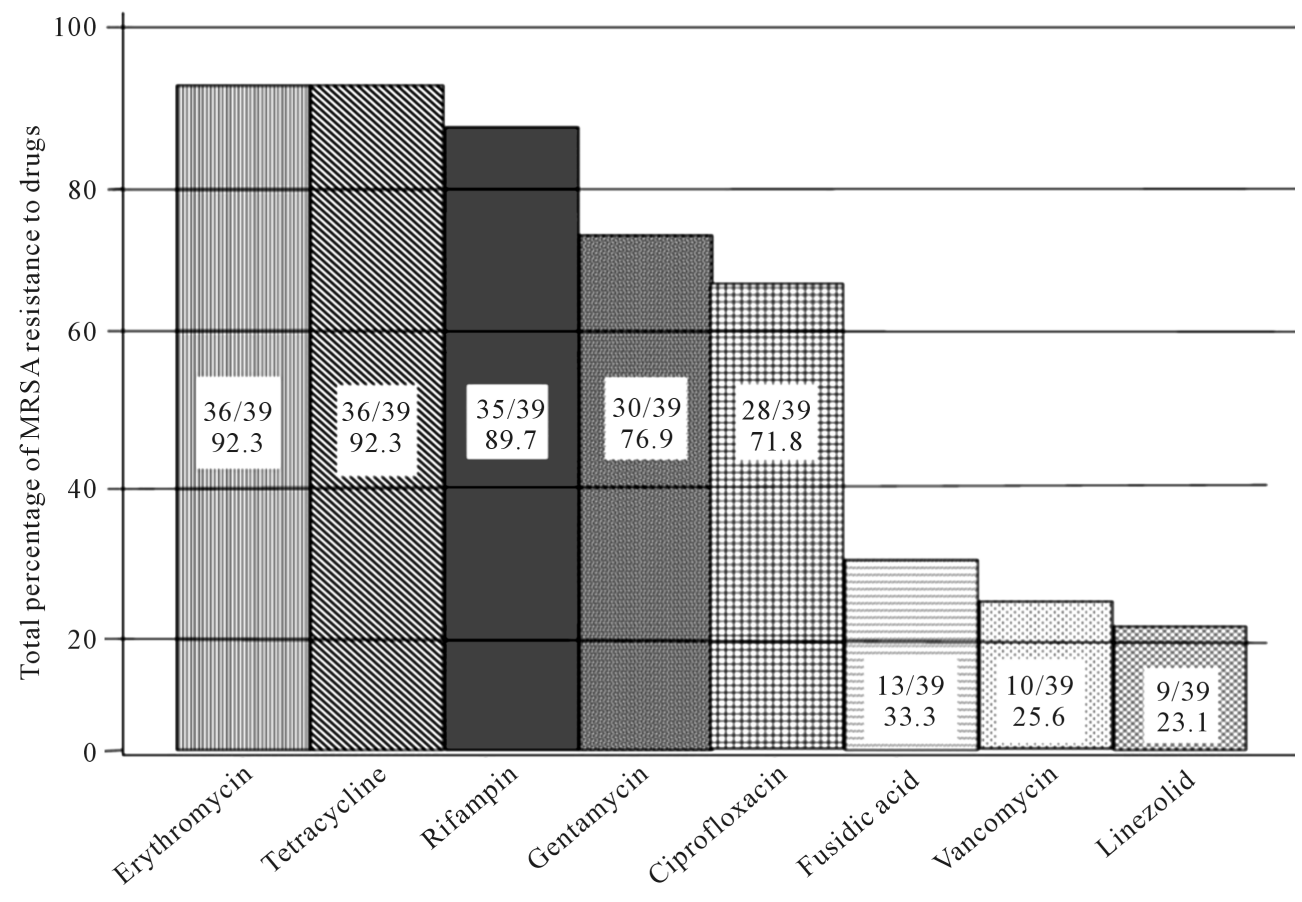

Figure 2. Resistance of MRSA to different anti-biograms.

The study has also shown that the MRSA is multi-drug resistant, a trend which has been demonstrated by other studies around the world [20]-[22]. There was significant resistance to anti-microbials that have been the bedrock of therapy in this country including gentamycin, tetracycline, ciprofloxacin, and erythromycin. Gentamycin showing moderate resistance of $66 \%$ could still be considered for use in the treatment of certain strains, at high dose. Erythmycin and tetracycline should however not be considered at all due to high resistance at 92\%. There was significant clindamycin resistance as well as shown by the positive D-test at $74 \%$. This inducible resistance has also been shown by other studies [23]-[25].

Multi-drug resistant MRSA is difficult to treat and has been identified as one of the most serious threats to health in this century [26]. For developing countries like Kenya, this spells disaster to efforts to secure health. The efficacious anti-microbials shown in this study like linezolid with sensitivity of $72 \%$, vancomycin at $70 \%$ and fucidic acid at $67 \%$, push the standard of treatment beyond affordable means of the majority.

The areas of hospital that need close monitoring include, surgical wards, burns unit, renal unit and intensive care unit where higher yields of MRSA were isolated. Considering that there are limited anti-microbial options in MTRH, wide spread occurrence of MRSA will lead to high mortality, morbidity, and increased cost of treatment and length of hospital stay. These are real challenges for a resource-constrained region. Continuous drug resistance surveillance, prudent use of anti-microbials and above all infection control measures are needed to stem the tide of MRSA spread.

\section{Conclusion}

The study showed that MRSA is present at MTRH, most of which was from pus, tracheal aspirates and urine. The units with high burden of MRSA were Medical wards, surgical wards and ICU. Multidrug-resistance was evident and only limited number of anti-microbials can effectively treat MRSA in MTRH. Inducible clindamycin resistance was also shown to be significantly high.

\section{Acknowledgements}

I thank the MTRH management for allowing me conduct the study in the institution. I also thank IREC for the study approval and MTRH Microbiology Staff for their moral support. 


\section{References}

[1] Diekman, D.J. and Pfaller, M.A. (2000) Genetic Relatedness of Multidrug-Resistant, Methicillin (Oxacillin)-Resistant Staphylococcus aureus Bloodstream Isolates form SENTRY Antimicrobial Resistance Surveillance Centers Worldwide, 1998. Microbial Drug Resistance, 6, 213-221. http://dx.doi.org/10.1089/mdr.2000.6.213

[2] Cosgrove, S.E., Qi, Y., Kaye, K.S., Harbarth, S., Karchmer, A.W. and Carmeli, Y. (2005) The Impact of Methicillin Resistance in Staphylococcus aureus Bacteremia on Patient Outcomes: Mortality, Length of Stay and Hospital Charges. Infection Control and Hospital Epidemiology, 26, 166-174. http://dx.doi.org/10.1086/502522

[3] Whitby, M., McLaws, M.L. and Berry, G. (2001) Risk of Death from Methicillin-Resistant Staphylococcus aureus Bacteraemia: A Meta-Analysis. The Medical Journal of Australia, 175, 264-267.

[4] Kesah, C., Ben Redjeb, S., Odugbemi, T.O., Boye, C.S., Dosso, M., Ndinya Achola, J.O., Koulla-Shiro, S., Benbachir, M., Rahal, K. and Borg, M. (2003) Prevalence of Methicillin Resistant Staphylococcus aureus in Eight African Hospitals and Malta. Clinical Microbiology and Infection, 9, 153-156. http://dx.doi.org/10.1046/j.1469-0691.2003.00531.x

[5] Shittu, A.O. and Lin, J. (2006) Antimicrobial Susceptibility Patterns and Characterization of Clinical Isolates of Staphylococcus aureus in Kwazulu-Natal Province, South Africa. BMC Infectious Diseases, 6, 125. http://dx.doi.org/10.1186/1471-2334-6-125

[6] Kirby, W.M.M. (1944) Extraction of a Highly Potent Penicillin Inactivator from Penicillin Resistant Staphylococcus. Science, 99, 452-453. http://dx.doi.org/10.1126/science.99.2579.452

[7] Roundtree, P.M. and Freeman, B.M. (1956) Infections Caused by a Particular Phage Type of Staphylococcus aureus. The Medical Journal of Australia, 42, 157-161.

[8] Jevons, M. (1961) “Celbenin”-Resistant Staphylococcus. British Medical Journal, 1, 124-125. http://dx.doi.org/10.1136/bmj.1.5219.124-a

[9] Chambers, H.F. (2005) Community-Associated MRSA-Resistance and Virulence Converge. The New England Journal of Medicine, 352, 1485-1487. http://dx.doi.org/10.1056/nejme058023

[10] Changtrakool, P., Ito, T., Ma, X.X., et al. (2006) Staphylococcal Cassette Chromosome mec (SCCmec) Typing of Methicillin-Resistant Staphylococcus aureus Strains Isolated in Asian Countries: A Proposal for New Nomenclature for SCCmec Elements. Antimicrobial Agents and Chemotherapy, 50, 1001-1012. http://dx.doi.org/10.1128/AAC.50.3.1001-1012.2006

[11] Oliveira, D.C., Tomasz, A. and de Lancastre, H. (2001) The Evolution of Pandemic Clones of Methicillin-Resistant Staphylococcus aureus Identification of Two Ancestral Genetic Backgrounds and the Associated mec Elements. Microbial Drug Resistancet, 7, 349-361. http://dx.doi.org/10.1089/10766290152773365

[12] Zhang, H.Z., Hackbarth, C.J., Chanksky, K.M. and Chambers, H.F. (2001) A Proteolytic Transmembrane Signaling Pathway and Resistance to Beta-Lactams in Staphylococci. Science, 291, 1965. http://dx.doi.org/10.1126/science.1055144

[13] Mehta, A.P., Rodrigues, C., Sheth, K., Jani, S., Hakimiyan, A. and Fazalbhoy, N. (1998) Control of Methicillin Resistant Staphylococcus aureus in a Tertiary Care Centre-A Five-Year Study. Indian Journal of Medical Microbiology, 16, 31-34.

[14] DeLeo, F.R. and Chambers, H.F. (2009) Reemergence of Antibiotic-Resistant Staphylococcus aureus in the Genomics Era. Journal of Clinical Investigation, 119, 2464-2474. http://dx.doi.org/10.1172/JCI38226

[15] Klevens, R.M., Morrison, M.A. and Nadle, J. (2007) Active Bacterial Core Surveillance (ABCs) MRSA Investigators. Invasive Methicillin-Resistant Staphylococcus aureus Infections in the United States. JAMA, 298, 1763-1771. http://dx.doi.org/10.1001/jama.298.15.1763

[16] Swenson, J.M. and Tenover, F.C. (2005) Results of Disk Diffusion Testing with Cefoxitin Correlate with Presence of mecA in Staphylococcus spp. Journal of Clinical Microbiology, 43, 3818-3823. http://dx.doi.org/10.1128/JCM.43.8.3818-3823.2005

[17] Clinical and Laboratory Standard Institute (CLSI) (2010) Performance Standards for Antimicrobial Susceptibility Testing; Twentieth Informational Supplement. CLSI Document M100-S20. Wayne, Pennsylvania.

[18] Mwambu, O.T., Jolobo, M., Agwu, E., Bwanga, F., Najjuka, C. and Kaddu-Mulindwa, D. (2008) Prevalence of Methicillin Resistant Staphylococcus aureus (MRSA) among Isolates from Surgical Site Infections in Mulago HospitalKampala, Uganda. The Internet Journal of Infectious Diseases, 7, 3.

[19] Kobayashi, H. (2005) National Hospital Infection Surveillance on Methicillin-Resistant Staphylococcus aureus. Journal of Hospital Infection, 60, 172-175. http://dx.doi.org/10.1016/j.jhin.2004.12.010

[20] Kim, H.B., Jang, H.-C., Nam, H.J., Lee, Y.S., Kim, B.S., Park, W.B., Lee, K.D., Choi, Y.J., Park, S.W., Oh, M.D., Kim, E.-C. and Choe, K.W. (2004) In-Vitro Activities of 28 Antimicrobial Agents against Staphylococcus aureus Iso- 
lates from Tertiary-Care Hospitals in Korea: A Nationwide Survey. Antimicrobial Agents and Chemotherapy, 48, 1124-1127. http://dx.doi.org/10.1128/AAC.48.4.1124-1127.2004

[21] Fluit, A.C., Wielders, C.L.C., Verhoef, J. and Schmitz, F.J. (2001) Epidemiology and Susceptibility of 3,051 Staphylococcus aureus Isolates from 25 University Hospitals Participating in the European SENTRY Study. Journal of Clinical Microbiology, 39, 3727-3732. http://dx.doi.org/10.1128/JCM.39.10.3727-3732.2001

[22] Santos Sanches, I.S., Mato, R., de Lencastre, H. and Tomasz, A., CEM/NET Collaborators (2000) The International Collaborators Patterns of Multidrug Resistance among Methicillin-Resistant Hospital Isolates of Coagulase-Positive and Coagulase-Negative Staphylococci Collected in the International Muticenter Study Resist in 1997 and 1998. Microbial Drug Resistance, 6, 199-211. http://dx.doi.org/10.1089/mdr.2000.6.199

[23] Fiebelkorn, K.R., Crawford, S.A., McElmeel, M.L. and Jorgensen, J.H. (2003) Practical Disk Diffusion Method for Detection of Inducible Clindamycin Resistance in Staphylococcus aureus and Coagulase-Negative Staphylococci. Journal of Clinical Microbiology, 41, 4740-4744. http://dx.doi.org/10.1128/JCM.41.10.4740-4744.2003

[24] LaPlante, K.L., Rybak, M.J., Amjad, M. and Kaatz, G. W. (2007) Antimicrobial Susceptibility and Staphylococcal Chromosomal Cassette mec Type in Community- and Hospital-Associated Methicillin-Resistant Staphylococcus aureus. Pharmacotherapy, 27, 3-10. http://dx.doi.org/10.1592/phco.27.1.3

[25] Siberry, G.K., Tekle, T., Carroll, K. and Dick, J. (2003) Failure of Clindamycin Treatment of Methicillin-Resistant Staphylococcus aureus Expressing Inducible Clindamycin Resistance in Vitro. Clinical Infectious Diseases, 37, 12571260. http://dx.doi.org/10.1086/377501

[26] Smolinski, M.S., Hamburg, M.A. and Lederberg, J., Eds. (2003) Microbial Threats to Health: Emergence, Detection, and Response. Institute of Medicine, Washington, 32. 